\title{
Exposure of cats to low doses of FeLV: seroconversion as the sole parameter of infection
}

\author{
Andrea MAJor ${ }^{1}$, Valentino CATTORI ${ }^{1 *}$, Eva Boenzli ${ }^{1}$, Barbara Riond ${ }^{1}$, \\ Peter Ossent ${ }^{2}$, Marina Luisa Meli ${ }^{1}$, Regina Hofmann-Lehmann ${ }^{1}$, Hans Lutz ${ }^{1}$ \\ ${ }^{1}$ Clinical Laboratory, Vetsuisse Faculty, University of Zurich, Winterthurerstrasse 260, 8057, Zurich, Switzerland \\ ${ }^{2}$ Institute of Veterinary Pathology, Vetsuisse Faculty, University of Zurich, Winterthurerstrasse 268, 8057, \\ Zurich, Switzerland
}

(Received 15 July 2009; accepted 26 October 2009)

\begin{abstract}
In felids, feline leukemia virus (FeLV) infection results in a variety of outcomes that range from abortive (virus readily eliminated and never detectable) to progressive infection (persistent viremia and viral shedding). Recently, a novel outcome was postulated for low FeLV infectious doses. Naïve cats exposed to faeces of persistently infected cats seroconverted, indicating infection, but remained negative for provirus and p27 antigen in blood. FeLV provirus was found in some tissues but not in the bone marrow, infection of which is usually considered a necessary stage for disease progression. To investigate the impact of low FeLV doses on young cats and to test the hypothesis that low dose exposure may lead to an unknown pathogenesis of infection without involvement of the bone marrow, 21 cats were infected oronasally with variable viral doses. Blood p27, proviral and viral loads were followed until week 20 post-infection. Tissue proviral loads were determined as well. The immune response was monitored by measuring FeLV whole virus and p45 antibodies; and feline oncornavirus-associated cell membrane antigen (FOCMA) assay. One cat showed regressive infection (transient antigenemia, persistent provirus-positivity, and seroconversion) with provirus only found in some organs at sacrifice. In 7 of the 20 remaining cats FOCMA assay positivity was the only sign of infection, while all other tests were negative. Overall, the results show that FeLV low dose exposure can result in seroconversion during a presumed abortive infection. Therefore, commonly used detection methods do not detect all FeLV-infected animals, possibly leading to an underestimation of the prevalence of infection.
\end{abstract}

\section{FeLV / pathogenesis / infection outcome / abortive infection / FOCMA assay}

\section{INTRODUCTION}

Feline leukemia virus (FeLV) is a retrovirus of great veterinary importance that was discovered more than 40 years ago [20] and infects domestic cats and some related small felids worldwide [18, 22, 32]. An infection with FeLV may cause disorders of hematopoietic cells, a state of immunodeficiency and fatal neoplasia. Common symptoms are fever, anaemia, anorexia and weight loss. The prevalence of FeLV

\footnotetext{
*Corresponding author: vcattori@vetclinics.uzh.ch
}

infection has been decreasing in the past few years. In Switzerland, FeLV prevalence was found to be $3 \%$ in healthy and of up to $13 \%$ in ill cats [29]. Decreasing prevalence is the consequence of identification and segregation of infected cats, and of extensive vaccination programs. However, cases of recurrence keep occurring and emphasise the importance of an accurate diagnosis [24]. FeLV is mainly transmitted directly from cat to cat. Transmission occurs through contact with saliva via licking, mutual grooming and sharing of food or water dishes or trough bites [5, 6]. Infectious FeLV 
can also be transmitted via faeces and milk $[8,34]$. FeLV RNA was also detected in urine, but, its infection potential has not been demonstrated so far [1]. Pathogenesis is well understood if infectious pressure is high. Infection starts in the oropharynx where the virus first replicates in tonsils and local lymph nodes [37], from which the virus spreads to the bone marrow, thymus, spleen and intestine through infected lymphocytes. Bone marrow infection is an important hallmark of FeLV pathogenesis as the virus replicates extensively in bone marrow and infects blood precursor cells first, and many organs and tissues thereafter, including salivary gland, tonsils, pharyngeal, urinary bladder, gastric, intestinal, colonic, pancreatic and endometrial epithelia, lymph nodes throughout the body, bone marrow, and spleen $[36,37]$. FeLV infection presents a variety of outcomes [11, 16-18, 25, 27, 35, 37, 38], which are influenced by both host and virus factors. Known host resistance factors include age and immune system status. Known virologic factors are virus strain, subtype and viral infection dose [18]. The outcome of infection is still a rather controversial issue. In the past, infection outcome was classified as viremic, non-viremic, and transiently viremic based on results of virus isolation, immunofluorescence assays and/or antigen detection [27]. Viremic cats are characterized by continuous expression of p27 viral antigen. FeLV infection is not contained due to a lack of FeLV specific immunity $[3,4,18]$. Viremic cats continuously shed virus, thereby posing a risk of infection to susceptible cats, and usually succumb to FeLV-associated diseases (anaemia, immunosuppression, and neoplasia). In transiently viremic cats, viremia is overcome after a few weeks post-infection (p.i.). However, transiently viremic cats remain provirus positive [15]. In addition, some nonviremic cats were shown to have localized infection characterized by virus replication in certain tissues, such as mammary, salivary and urinary epithelium $[8,12,34]$. This additional form of FeLV infection was termed atypical or sequestered infection. New sensitive molecular assays have been described recently for the use in detection and quantification of FeLV provirus DNA and viral RNA [14, 39, 42], resulting in a more sensitive measure for FeLV exposure. The spectrum of host response categories was refined accordingly. Especially in p27 negative cats, the outcome should be reevaluated based on the presence or absence of FeLV proviral DNA in blood or bone marrow. The spectrum of host response categories was thus reclassified into: abortive (no virus detected after exposure), regressive (p27-negative, provirus positive after or without transient antigenemia) and progressive (persistently p27 positive, virus isolation, provirus positive, FeLV RNA positive) [16]. Whether cats that show no signs of infection (abortive) are truly immune or just resistant, is still open to debate. In this context, a very interesting finding was that FeLV infection can be transmitted by contact with faeces [8] with no apparent viremia and infection involving the bone marrow, and constant negativity for proviral DNA in blood - which would be classified as abortive infection. However, in the study of GomesKeller et al. [8] FeLV provirus could be detected in several organs and the cats showed seroconversion, indicating that infection indeed had occurred. It was concluded that under low infectious pressure a different pathogenesis may take place in which bone marrow is not involved. Since FeLV transmission at very low infection levels appears to be the most natural way of infection, many cats may show this yet uncharacterized outcome. Cats that test negative for FeLV by conventional diagnostic methods could still have been infected and constitute a relevant portion of the cat population affected by the virus. The prevalence of FeLV infection may therefore have been underestimated, a factor that can have important consequences for FeLV control management, e.g. in Iberian lynxes, which seem to be particularly susceptible for FeLV infection and for which a correct estimation of FeLV spreading potential is of great importance [32]. It was thus the aim of this study to further characterize the course of infection after exposure to low infectious pressure and to test the hypothesis that low FeLV doses applied to young cats may lead to seroconversion even when the infection does not progress through bone marrow. The virus may not be completely eliminated and a limited 
viral replication in tissues may lead to a development of a weak immune response. Three groups of 7 cats were exposed to different, low doses of FeLV. Blood p27 antigen, proviral DNA and viral RNA were measured in blood at regular intervals until week 20 p.i. and in popliteal and mesenteric lymph nodes, bone marrow, spleen, kidney, urinary bladder, lungs, thymus, myocardium, parotid gland, and pancreas after euthanasia. Immune response against FeLV was assessed by the detection of antibodies by ELISA to FeLV whole virus and to FeLV p45 (the recombinant env-gene product), by immunofluorescence assay to feline oncornavirus-associated cell membrane antigen (FOCMA), and by Western blot analysis to the separated FeLV components.

\section{MATERIALS AND METHODS}

\subsection{Animals}

Twenty-six, 9 weeks-old Specific Pathogen-Free (SPF) male kittens were obtained from Liberty Research, Inc. (Waverly, NY, USA). Animals were kept under barrier conditions and under optimal ethological and hygienic conditions in one group of 5 cats (uninfected control group) and three groups of 7 cats each (group $1 \mathrm{~K}, 10 \mathrm{~K}$, and $100 \mathrm{~K}$, based on viral infectious dose). Prior to the beginning of the experiment the cats were tested by PCR, RT-PCR and serology and shown to be negative for FeLV, FIV, Herpes-, Corona-, Calici-, Parvovirus and feline hemotropic mycoplasmas.

\subsection{FeLV-A virus challenge}

At the age of 16 weeks, each kitten of groups $1 \mathrm{~K}$, $10 \mathrm{~K}$, and $100 \mathrm{~K}$ were infected once oronasally with FeLV-A/Glasgow-1 [19] by introducing $0.2 \mathrm{~mL}$ of virus suspension into each nostril and $0.6 \mathrm{~mL}$ into the mouth. The virus suspension for group $1 \mathrm{~K}$ contained a total of 1000 focus-forming units (FFU), the one for group $10 \mathrm{~K} 10000 \mathrm{FFU}$ and group $100 \mathrm{~K}$ $1000000 \mathrm{FFU}$. The viral stock origin and infectivity was the same as described in earlier works $[15,41]$.

\subsection{Sample collection and processing}

Blood samples were collected under sedation $\left(0.01 \mathrm{mg} / \mathrm{kg}\right.$ midazolam (Dormicum ${ }^{\circledR}$, Roche Pharma
AG, Reinach, Switzerland) and $10 \mathrm{mg} / \mathrm{kg}$ ketamine (Narketan ${ }^{\circledR}$, Vétoquinol AG, Belp, Switzerland)) prior to challenge at week $-7,-5,-3$, and then weekly, starting from week 0 until week 6 p.i., later in biweekly intervals until week 20 p.i. Blood samples were obtained by jugular venipuncture using $5 \mathrm{~mL}$ syringes and blood was immediately transferred into EDTAtubes. $400 \mu \mathrm{L}$ of EDTA blood was submitted for haematology analysis, $200 \mu \mathrm{L}$ of EDTA anticoagulated whole blood were aliquoted for DNA extraction. Plasma was obtained by centrifuging approximately $2 \mathrm{~mL}$ of EDTA blood at $1700 \times g$ for $10 \mathrm{~min}$. Blood and plasma samples were immediately frozen at $-80{ }^{\circ} \mathrm{C}$ until they were processed.

\subsection{Detection of proviral DNA and plasma viral RNA}

For determination of FeLV proviral loads, total nucleic acids were extracted from a blood volume containing $10^{6}$ white blood cells using the MagNa Pure LC Total Nucleid Acid Isolation Kit (Roche Diagnostics AG, Rotkreuz, Switzerland). The extracted total nucleic acids were analyzed by real-time TaqMan PCR as described in [39] using the $2 \times$ TaqMan ${ }^{\mathbb{B}}$ Fast Universal PCR Master Mix (Applied Biosystems, Foster City, CA, USA) on a ABI 7500 sequence detection system (Applied Biosystems) and under the following cycling conditions: an initial denaturation of $20 \mathrm{~s}$ at $95{ }^{\circ} \mathrm{C}$ was followed by 45 cycles of $95^{\circ} \mathrm{C}$ for $3 \mathrm{~s}$ and $60{ }^{\circ} \mathrm{C}$ for $30 \mathrm{~s}$. For each run, a glyceraldehyde-3-phophate dehydrogenase (GAPDH) pseudogene of which one copy is present in the genomic DNA of feline cells [33] was also quantified as described [39] using the $2 \times$ TaqMan $^{(B)}$ Fast Universal PCR Master Mix and the same PCR run conditions as for FeLV provirus. FeLV proviral DNA amounts were normalized to feline GAPDH by dividing FeLV copy numbers by fGAPDH copy numbers to calculate FeLV copies per cell. Viral RNA in plasma samples was extracted from $200 \mu \mathrm{L}$ of plasma (either from 5-sample pools or from single samples) using the MagNa Pure LC Total Nucleic Isolation Kit and quantified by real-time TaqMan reverse transcriptase (RT)-PCR as described [39] using a ABI 7500 sequence detection system.

\subsection{Detection of FeLV virus protein p27 by ELISA}

The presence of plasma FeLV p27 antigen was determined using a sandwich ELISA as previously described [26]. Results are represented as percentages of a defined positive control (culture supernatant of 
FL-74 feline lymphoblastoid cell line permanently expressing FeLV), which was considered $100 \%$. Samples reaching $>5 \%$ of the positive control signal were considered positive [14].

\subsection{Antibody assays}

The plasma samples were also analysed for the presence of antibodies to FeLV whole virus, to FeLV p45 (the non-glycosylated form of gp70 surface unit of the envelope glycoprotein), and to FOCMA. Anti-FeLV p45 and anti-FeLV whole virus antibodies were measured by ELISA as described [21, 25], using $100 \mathrm{ng}$ of p45/well and $100 \mathrm{ng}$ of gradient purified FL-74 FeLV, respectively. Plasma was used at a dilution of 1:200 and antibody levels assessed by comparison with predefined control antisera [25]. Antibody to FOCMA was measured at week 0 and week 20 p.i., by indirect cell membrane immunofluorescence as described [2]. FL-74 cell culture medium was tested for the absence of FCV, FHV, FPV, FCoV, FIV, hemotropic mycoplasma and presence of FeLV by RT-PCR/PCR as described $[9,13,23,31,39,44,45]$. The culture was consistently free of the unwanted contaminants. The cat sera were titrated at 4-fold dilutions from 1:4 to 1:256. Samples showing a minimal titre of $1: 4$ were considered to be FOCMA positive. In addition, samples from week -3 and week 20 p.i. were examined for the presence of antibodies to FeLV gp70, p27 and p15(E) $[26,30]$ by Western blot analysis as described [28].

\subsection{Detection of FeLV provirus and virus isolation from tissues and bone marrow}

Cats of group $10 \mathrm{~K}$ and group $100 \mathrm{~K}$ were euthanized at week 20 , and tissue samples from popliteal and mesenteric lymph nodes, bone marrow, spleen, kidney, urinary bladder, lungs, thymus, myocardium, parotid gland, and pancreas were collected within 30 min post-mortem. Samples were snap-frozen in liquid nitrogen. Ten $\mathrm{mg}$ of tissue were used for the extraction of DNA using MagNA Pure LC DNA Isolation Kit II (Tissue kit, Roche Diagnostics). FeLV provirus was quantified by real-time PCR as described $[39,40]$. To guarantee a comparable sensitivity, samples yielding less than 15000 fGAPDH copies per reaction were re-extracted until a suitable concentration was reached. In addition, samples were collected under sterile conditions from mesenteric lymph node, urinary bladder, lungs, thymus, and myocardium for virus isolation. Tissue samples were co-cultured with FEA cells and supernatants of the co-cultures collected at days $4,8,12,16,20,24$, and 28 post-inoculation were tested for $\mathrm{p} 27$ antigen by ELISA as described [7]. Bone marrow samples were cultured in medium and supernatants collected at days 18 and 21 were used for total nucleic acid isolation (MagNA Pure LC Total Nucleic Acid Isolation Kit, Roche Diagnostics) and samples were tested for the presence of FeLV RNA. Additionally, supernatants were tested by p27 ELISA [26].

\subsection{Statistics}

Statistical analyses were carried out using $\mathrm{R}$ software version 2.9.0. (The $\mathrm{R}$ Foundation for Statistical Computing, Vienna, Austria). Longitudinal effects (time) on antibody titres of the different groups were compared to each other by multivariate analysis of variance (MANOVA). A $p$ value $<0.05$ was considered to be statistically significant.

\section{RESULTS}

\subsection{FeLV DNA and RNA detection in blood}

With one exception, cats did not become provirus positive at any time point tested. The one cat (JCR2) that was found to be positive belonged to the group challenged with the highest of the three doses of FeLV (100 000 FFU). Proviral DNA was detected from week 2 p.i. on with a peak at week 3 (3.33 copies/cell) (Fig. 1). Similarly, viral RNA was consistently detected only in samples of the provirus-positive cat (JCR2) until week 12 and additionally at week 18 once more. The highest viral RNA load measured was at week 2 p.i. (Ct-value of 22.95, Fig. 1). Viral RNA was not detectable in pooled plasma samples (5 samples each pool) of any of the provirus-negative cats at week 0,10 , and 20 p.i. In addition, samples from week 2,3 , and 4 were tested individually, as the viral RNA peak usually occurs in this time period; the results were negative, too.

\subsection{Detection of p27 in plasma}

p27 antigen was detected in plasma of one (JCR2) of the 26 cats tested. This cat became transiently positive from week 3 p.i. to week 4 p.i. (Fig. 1). p27 was not detectable at any time point in all other cats. 


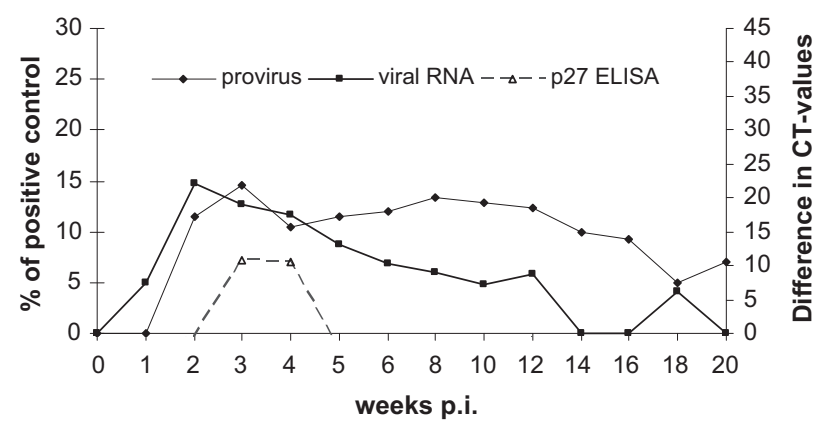

Figure 1. Proviral DNA, viral RNA, and plasma p27 loads from the persistent PCR-positive cat JCR2 measured at regular intervals until week 20 p.i. Provirus and virus loads are expressed as 45 minus the cycle threshold $(\mathrm{Ct})$ values measured. p27 results are represented as percentage in comparison to a positive control (FL-74 lymphoblastoid cell culture supernatant), which was considered to be $100 \%$. Cat JCR2, found to be consistently provirus positive, showed a regressive infection with transient antigenemia.

\subsection{Antibody assays}

By measuring antibodies against FeLV p45 and whole virus by ELISA, seroconversion was observed in one cat. In the p45 ELISA, this cat (JCR2) had a positive response throughout weeks 8 to 20 with a peak of $26 \%$ of the positive control at week 14 , and throughout weeks 4 to 20 with a peak of $54 \%$ of the positive control at week 20 in the FeLV whole virus ELISA (data not shown). Analysis of the remaining animals showed no statistically relevant difference between the groups in p45 as well as whole virus ELISA (P $\mathrm{P}_{\text {MANOvA }} 0.53$ and 0.47 , respectively Figs. 2 and 3). Prior to challenge, all samples were negative for the presence of antibodies directed against FOCMA. At week 20 p.i., antibodies to FOCMA were detected in 4 cats (KCT1, JCU3, JCO2, JCP1, 57\%) of group $10 \mathrm{~K}$ and 4 (KDA1, JCR2, KCQ1, $\mathrm{JCS} 1,57 \%$ ) cats of group $100 \mathrm{~K}$. Of these 8 cats, the PCR-positive cat (JCR2) reached the highest titre (1:64), the other 7 cats developed antibody titres which ranged from 1:4 to $1: 16$. No negative control group and group $1 \mathrm{~K}$ animal had a detectable titre (Tab. I). In Western blot analysis, sera from 2 cats of group $10 \mathrm{~K}$ (JCU3, JCS2) showed a weak reactivity to the upper band of the $\mathrm{p} 15(\mathrm{E})$ protein at a serum dilution of 1:40 as well as at 1:100. However, the same cats showed some reactivity to the upper band of p15(E) already at week 0 p.i. (Fig. 4). In the PCR-positive cat (JCR2) the presence of antibodies against FeLV was confirmed at a dilution of 1:100. All other cats from group $100 \mathrm{~K}, 10 \mathrm{~K}$ and $1 \mathrm{~K}$ had either a completely negative Western blot pattern or showed cross-reactive bands already present at week -3 (cats KDA1 and JCS2, Fig. 4).

\subsection{Detection of FeLV DNA sequences and virus isolation from tissues}

At week 20 post-challenge, cats of groups $10 \mathrm{~K}$ and $100 \mathrm{~K}$ were euthanized and different tissue samples were collected and analysed for the presence of FeLV DNA sequences. Popliteal and mesenteric lymph nodes, bone marrow, spleen, kidney, urinary bladder, lungs, thymus, myocardium, parotid gland, and pancreas were tested. FeLV DNA sequences were found in organs of the cat (JCR2) positive for FeLV provirus in blood. The copy numbers were in average very low and ranged from 5.6 to 704.25 copies/reaction $\left(3.4 \times 10^{-5}\right.$ to 0.0024 copies/cell, data not shown). The popliteal lymph node showed the highest FeLV proviral load ( 0.0024 copies/cell). No FeLV DNA sequences were detected in tissue samples of all other cats. Virus isolation from urinary bladder, myocardium, lungs, mesenteric lymph node, thymus, and bone marrow was negative for all cats. 


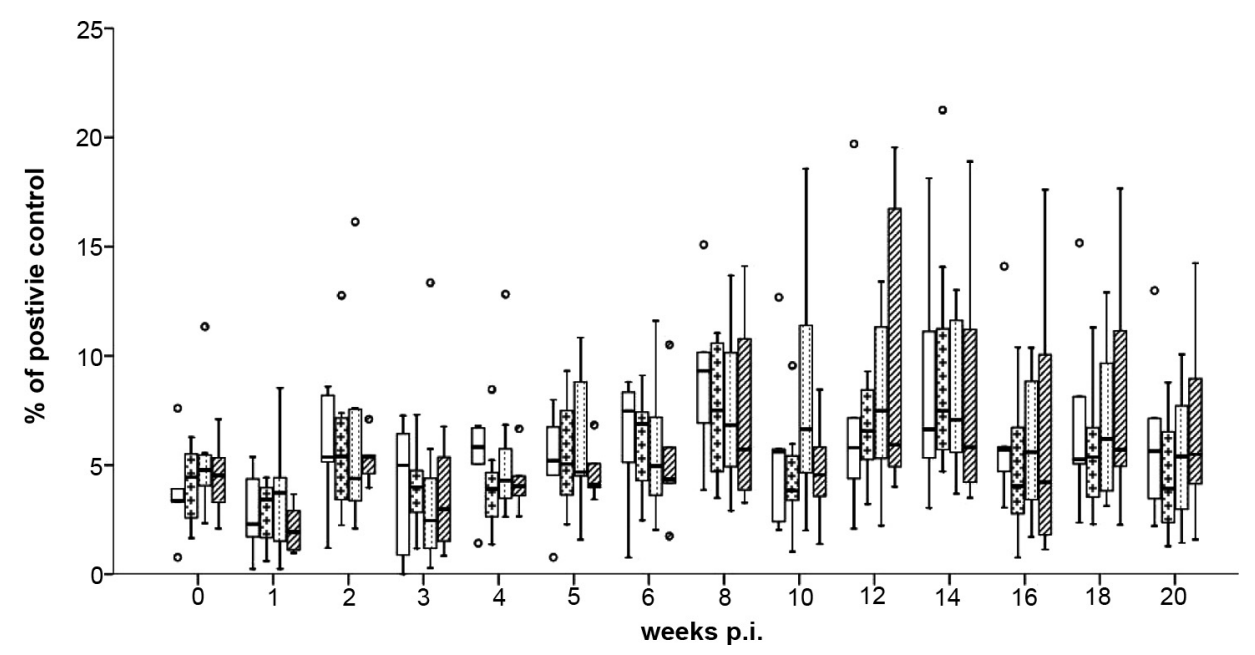

Figure 2. Box-plot results obtained measuring antibodies directed to FeLV p45 over the whole observation period as percentage of the positive control. The PCR-positive cat of group $100 \mathrm{~K}$ was excluded. No statistically relevant difference between the groups was observed. Open boxes: negative controls, crossed boxes: $1 \mathrm{~K}$ group, dotted boxes: $10 \mathrm{~K}$ group, hatched boxes: $100 \mathrm{~K}$ group. Outliers are depicted by a dot.

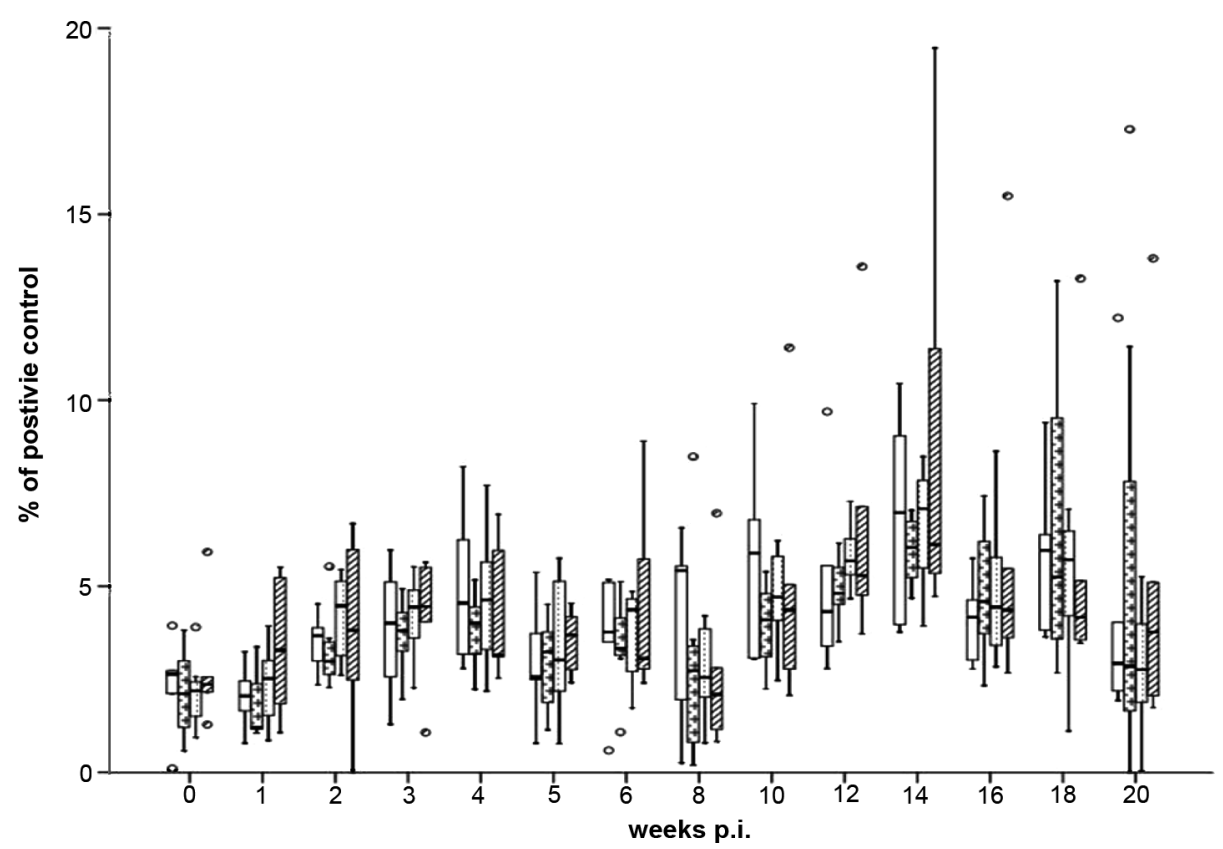

Figure 3. Antibodies directed to FeLV whole virus. Results are expressed as percentage of a positive control. The PCR-positive cat of group $100 \mathrm{~K}$ was excluded. No statistically relevant difference between the groups was observed. Open boxes: negative controls, crossed boxes: $1 \mathrm{~K}$ group, dotted boxes: $10 \mathrm{~K}$ group, hatched boxes: $100 \mathrm{~K}$ group. Outliers are depicted by a dot. 
Table I. Indirect FL-74 FOCMA immunofluorescence pre- and post-exposure test to low FeLV doses. Numbers of the titre column represent the antibodies titre, numbers in the groups columns represent the number of cats with the respective titre.

\begin{tabular}{|c|c|c|c|c|c|c|c|c|}
\hline \multirow[t]{2}{*}{ Titre } & \multicolumn{2}{|c|}{ Negative control } & \multicolumn{2}{|c|}{ Group $1 \mathrm{~K}$} & \multicolumn{2}{|c|}{ Group $10 \mathrm{~K}$} & \multicolumn{2}{|c|}{ Group $100 \mathrm{~K}$} \\
\hline & Week 0 & Week 20 & Week 0 & Week 20 & Week 0 & Week 20 & Week 0 & Week 20 \\
\hline Negative & 7 & 7 & 7 & 7 & 7 & 3 & 7 & 3 \\
\hline $1: 4$ & 0 & 0 & 0 & 0 & 0 & 2 & 0 & 3 \\
\hline $1: 16$ & 0 & 0 & 0 & 0 & 0 & 2 & 0 & 0 \\
\hline $1: 64$ & 0 & 0 & 0 & 0 & 0 & 0 & 0 & 1 \\
\hline
\end{tabular}

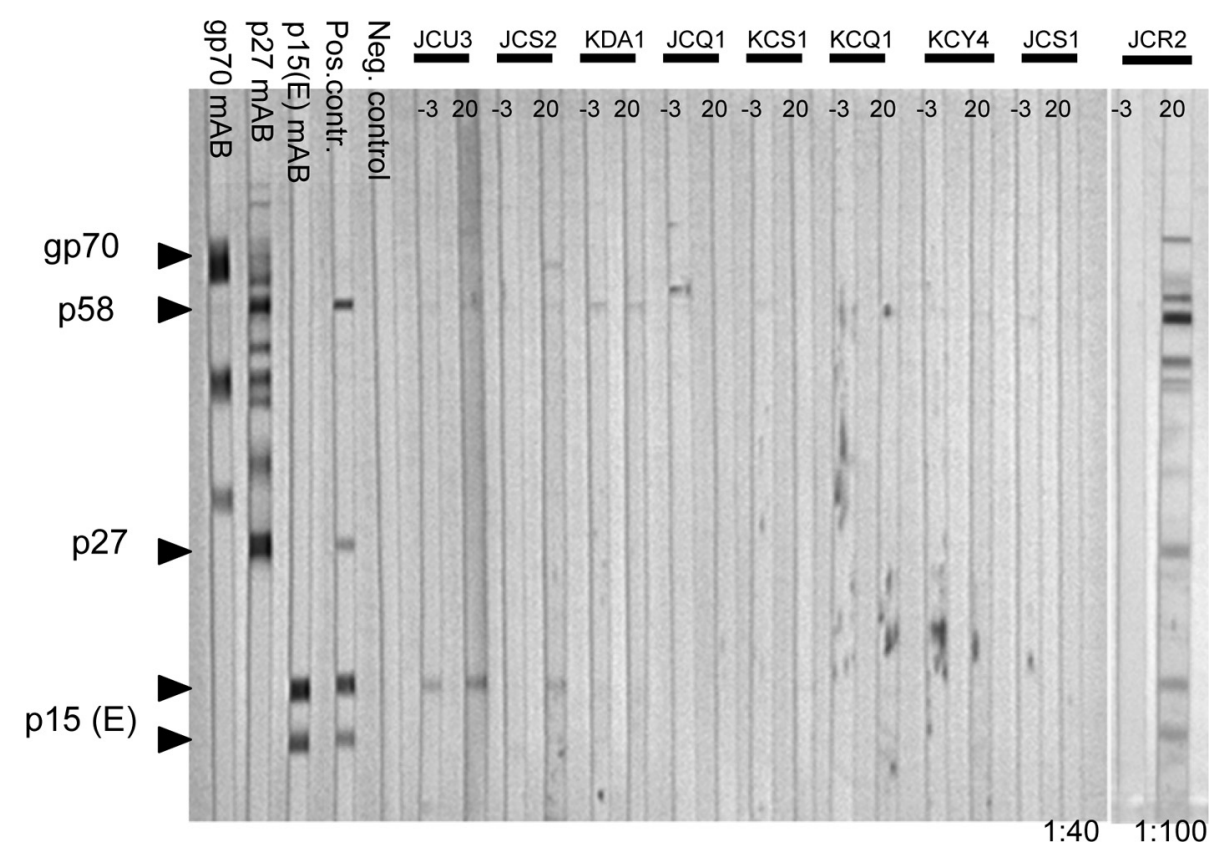

Figure 4. Western blot analysis using plasma samples from week -3 (prior to challenge) and week 20 p.i. (cats group $100 \mathrm{~K}$ and two of group $10 \mathrm{~K}$ ). The first 3 strips to the left were incubated with monoclonal antibodies against gp70, p27 and p15(E), respectively, to characterize the respective viral proteins. Serum obtained from a pool of immune cats used as a positive control. Cat JCU3 and cat JCS2 (group 10K) show a weak reaction with the upper band of the p15(E) protein. JCR2 shows antibodies to all FeLV proteins. In all other cats, responses to any FeLV proteins could not be detected. ( ) bands with expected length. All samples were tested under the same assay conditions.

\section{DISCUSSION}

The aim of this study was to investigate to what degree cats can become infected by low dose oral FeLV infection without detectable involvement of the bone marrow. Even though
FeLV prevalence has reportedly decreased during the past years, FeLV is still among the most important infectious diseases of cats. Hence, a deep knowledge of all aspects of FeLV pathogenesis and biology is essential. Recently, a novel course of infection was postulated that 
deviates from the classical course involving a phase in which bone marrow is infected. In this alternative course, cats exposed to low loads of virus via faecal route seroconverted without involvement of bone marrow and without showing any other sign of infection [8]. Under field conditions, exposure to small virus loads is expected to be frequent due to superficial direct contact with viremic cats shedding FeLV or indirectly through contact with excretions produced by viremic cats. Thus, many cats may show this newly recognized outcome. Since experimental infection had to mimic the natural mode of transmission trough faeces or saliva, in the present study we challenged the cats oronasally with viral loads that, based on published results [10] and on our experience, would induce abortive or regressive infection. Indeed, only one cat belonging to the group with the highest challenge dose showed regressive infection. For all other cats, abortive infection would have been assigned based on commonly used methods [16]. In this study, we demonstrated that a single oronasal exposure to FeLV as low as 10000 FFU is sufficient to elicit an antibody response and to lead to seroconversion in at least 18\% (lower 95\% confidence interval of the distribution) of the cats, demonstrating that the postulated alternative course of infection may occur in a considerable portion of the animals. We propose to designate this outcome of infection as abortive infection with seroconversion. No hallmark of infection was observed in the cats that received the smallest of the three doses (1 000 FFU), for which individual local innate immunity might have been enough to contain viral replication and to lead to abortive infection without a detectable adaptive immune response. However, under field conditions, multiple exposures in the range of $1000 \mathrm{FFU}$ or lower may occur giving rise to seroconversion or even provirus positivity. Considering that the assay for proviral DNA is able to detect one copy per reaction, we believe that the consistent PCR-negativity in blood of the cats that seroconverted is a strong indicator for the absence of bone marrow infection, a confirmation that an alternative course of infection may indeed exist, where the bone marrow is not involved [1]. FeLV provirus may still be integrated into the genome of the cells of some tissues but, in most cases, at levels beyond detection. In the study presented here, FeLV DNA sequences were not detectable in the organs tested. However, the presence of FeLV provirus in tissues other than the ones we analysed can not be excluded and should be investigated further. In localized infection where FeLV replication is confined to certain tissues, presence of plasma viral RNA loads is regarded a sensitive parameter for infection. RNA loads reflect ongoing viral replication somewhere in the cat's body even in sequestered places. However, detection of RNA is generally less frequent than that of proviral DNA [39]. Seroconversion was observed by use of the FL-74 cell membrane immunofluorescence test. This assay originally had been designated FOCMA test and was thought to detect antibodies at a non-virion tumour-associated antigen [2]. Later, it became clear that the FOCMA phenomenon can be explained not by a tumour specific antigen but by viral proteins including FeLV p15(E), gag-polyproteins and gp 70 of FeLV subtype C present on the FL-74 cell membrane [43]. The FL-74 cell membrane immunofluorescence test seems to be the most sensitive method to detect seroconversion compared to ELISA and Western blotting. This may be due in part to the less stringent test conditions, which allow antibodies with lower affinity to bind to their epitopes, and in part to the fact that in the FOCMA assay the binding targets are presented in a native conformation, thus allowing a better binding of conformation-specific antibodies. Induction of cytotoxic T-cells or virus-neutralizing antibodies by oral low dose infection, or Th1 cytokine expression that might have been induced by the experimental infection were not measured. Instead, antibody response was used as marker of infection. It is unclear to what degree the alternative pathway may be relevant to the spread of FeLV infection. We speculate that in most cases cats will overcome the infection for good. In very few cases - when the infected cats undergo immunosuppression due e.g. to concomitant FIV infection - full-blown FeLV infection with bone marrow involvement and viral shedding may develop. It will be important to determine to what extent this may occur. 
In conclusion, the present study provides additional evidence for the existence of an alternative FeLV infection course that leads to development of antibodies without involvement of the bone marrow. The results may be important for the surveillance of the FeLV status of a cattery or a cat population. Most likely a cattery cannot be considered free of FeLV as long as cats with FeLV-reactive antibodies are present. The potential for overt infection cannot be neglected.

Acknowledgements. We thank the technicians of the Clinical Laboratory, Vetsuisse Faculty, University of Zurich for excellent assistance in this project. Special thanks go to C. Braun, C. Robert, G. Wolf, V. Rüegger, and Y. Kessler for their helpful contributions and excellent support. The laboratory work was performed at the Centre of Clinical Studies from the Vetsuisse Faculty, University of Zurich, under the supervision of Dr M.L. Meli. This study was conducted by A. Major as fulfilment of the requirements for a doctoral thesis at the Vetsuisse Faculty, University of Zurich. R. Hoffmann-Lehmann is the recipient of a professorship from the Swiss National Science Foundation (PP00P3-119136). The authors are grateful for support by the United Bank of Switzerland (UBS) on behalf of a customer.

\section{REFERENCES}

[1] Cattori V., Pepin A.C., Tandon R., Riond B., Meli M.L., Willi B., et al., Real-time PCR investigation of feline leukemia virus proviral and viral RNA loads in leukocytes subsets, Vet. Immunol. Immunopathol. (2008) 123:124-128.

[2] Essex M., Klein G., Snyder S.P., Harrold J.B., Correlation between humoral antibody and regression of tumours induced by feline sarcoma virus, Nature (1971) 233:195-196.

[3] Flynn J.N., Hanlon L., Jarrett O., Feline leukaemia virus: protective immunity is mediated by virus-specific cytotoxic T lymphocytes, Immunology (2000) 101:120 125.

[4] Flynn J.N., Dunham S.P., Watson V., Jarrett O., Longitudinal analysis of feline leukemia virus-specific cytotoxic T lymphocytes: correlation with recovery from infection, J. Virol. (2002) 76:2306-2315.

[5] Francis D.P., Essex M., Excretion of feline leukaemia virus by naturally infected pet cats, Nature (1977) 269:252-254.

[6] Gomes-Keller M.A., Gonczi E., Tandon R., Riondato F., Hofmann-Lehmann R., Meli M.L., Lutz H., Detection of feline leukemia virus RNA in saliva from naturally infected cats and correlation of PCR results with those of current diagnostic methods, J. Clin. Microbiol. (2006) 44:916-922.
[7] Gomes-Keller M.A., Tandon R., Gonczi E., Meli M.L., Hofmann-Lehmann R., Lutz H., Shedding of feline leukemia virus RNA in saliva is a consistent feature in viremic cats, Vet. Microbiol. (2006) 112:11-21.

[8] Gomes-Keller M.A., Gonczi E., Grenacher B., Tandon R., Hofman-Lehmann R., Lutz H., Fecal shedding of infectious feline leukemia virus and its nucleic acids: a transmission potential, Vet. Microbiol. (2009) 134: 208-217.

[9] Gut M., Leutenegger C.M., Huder J.B., Pedersen N.C., Lutz H., One-tube fluorogenic reverse transcriptionpolymerase chain reaction for the quantitation of feline coronaviruses, J. Virol. Methods (1999) 77:37-46.

[10] Harbour D.A., Gunn-Moore D.A., Gruffydd-Jones T.J., Caney S.M., Bradshaw J., Jarrett O., Wiseman A., Protection against oronasal challenge with virulent feline leukaemia virus lasts for at least 12 months following a primary course of immunisation with Leukocell 2 vaccine, Vaccine (2002) 20:2866-2872.

[11] Hardy W.D. Jr, Hess P.W., MacEwen E.G., McClelland A.J., Zuckerman E.E., Essex M., et al., Biology of feline leukemia virus in the natural environment, Cancer Res. (1976) 36:582-588.

[12] Hayes K.A., Rojko J.L., Tarr M.J., Polas P.J., Olsen R.G., Mathes L.E., Atypical localised viral expression in a cat with feline leukaemia, Vet. Rec. (1989) 124:344-346.

[13] Helps C., Lait P., Tasker S., Harbour D., Melting curve analysis of feline calicivirus isolates detected by real-time reverse transcription PCR, J. Virol. Methods (2002) 106:241-244.

[14] Hofmann-Lehmann R., Huder J.B., Gruber S., Boretti F., Sigrist B., Lutz H., Feline leukaemia provirus load during the course of experimental infection and in naturally infected cats, J. Gen. Virol. (2001) 82:15891596.

[15] Hofmann-Lehmann R., Cattori V., Tandon R., Boretti F.S., Meli M.L., Riond B., et al., Vaccination against the feline leukaemia virus: outcome and response categories and long-term follow-up, Vaccine (2007) 25:5531-5539.

[16] Hofmann-Lehmann R., Cattori V., Tandon R., Boretti F.S., Meli M.L., Riond B., Lutz H., How molecular methods change our views of FeLV infection and vaccination, Vet. Immunol. Immunopathol. (2008) 124:119-123.

[17] Hoover E.A., Olsen R.G., Hardy W.D. Jr, Schaller J.P., Mathes L.E., Cockerell G.L., Biologic and immunologic response of cats to experimental infection with feline leukemia virus, Bibl. Haematol. (1975) 43:180-183.

[18] Hoover E.A., Mullins J.I., Feline leukemia virus infection and diseases, J. Am. Vet. Med. Assoc. (1991) 199:1287-1297.

[19] Jarrett O., Laird H.M., Hay D., Determinants of the host range of feline leukaemia viruses, J. Gen. Virol. (1973) 20:169-175.

[20] Jarrett W.F., Crawford E.M., Martin W.B., Davie F., A virus-like particle associated with leukaemia (lymphosarkoma), Nature (1964) 202:567-568. 
[21] Lehmann R., Franchini M., Aubert A., Wolfensberger C., Cronier J., Lutz H., Vaccination of cats experimentally infected with feline immunodeficiency virus, using a recombinant feline leukemia virus vaccine, J. Am. Vet. Med. Assoc. (1991) 199:1446-1452.

[22] Leutenegger C.M., Hofmann-Lehmann R., Riols C., Liberek M., Worel G., Lups P., et al., Viral infections in free-living populations of the European wildcat, J. Wildl. Dis. (1999) 35:678-686.

[23] Leutenegger C.M., Klein D., Hofmann-Lehmann R., Mislin C., Hummel U., Boni J., et al., Rapid feline immunodeficiency virus provirus quantitation by polymerase chain reaction using the TaqMan fluorogenic real-time detection system, J. Virol. Methods (1999) 78:105-116.

[24] Levy J., Crawford C., Hartmann K., HofmannLehmann R., Little S., Sundahl E., Thayer V., American Association of Feline Practitioners' feline retrovirus management guidelines, J. Feline Med. Surg. (2008) 10:300-316.

[25] Lutz H., Pedersen N., Higgins J., Hubscher U., Troy F.A., Theilen G.H., Humoral immune reactivity to feline leukemia virus and associated antigens in cats naturally infected with feline leukemia virus, Cancer Res. (1980) 40:3642-3651.

[26] Lutz H., Pedersen N.C., Durbin R., Theilen G.H., Monoclonal antibodies to three epitopic regions of feline leukemia virus p27 and their use in enzyme-linked immunosorbent assay of p27, J. Immunol. Methods (1983) 56:209-220.

[27] Lutz H., Pedersen N.C., Theilen G.H., Course of feline leukemia virus infection and its detection by enzyme-linked immunosorbent assay and monoclonal antibodies, Am. J. Vet. Res. (1983) 44:2054-2059.

[28] Lutz H., Arnold P., Hubscher U., Egberink H., Pedersen N., Horzinek M.C., Specificity assessment of feline T-lymphotropic lentivirus serology, Zentralbl. Veterinarmed. B (1988) 35:773-778.

[29] Lutz H., Lehmann R., Winkler G., Kottwitz B., Dittmer A., Wolfensberger C., Arnold P., Feline immunodeficiency virus in Switzerland: clinical aspects and epidemiology in comparison with feline leukemia virus and coronaviruses, Schweiz. Arch. Tierheilkd. (1990) 132:217-225 (in German).

[30] Lutz H.B.S., Corboz L., Müller R., Limacher W., Weber H., Wissler K., Theilen G.H., Combination of polyacrylamide gel electrophoresis with enzyme-linked immunosorbent assay: a simple method for the determination of antibody specifitiy and detection of antigens in complex mixtures, Vet. Immunol. Immunopathol. (1981) 2:425-440

[31] Meli M., Kipar A., Muller C., Jenal K., Gonczi E., Borel N., et al., High viral loads despite absence of clinical and pathological findings in cats experimentally infected with feline coronavirus (FCoV) type I and in naturally FCoV-infected cats, J. Feline Med. Surg. (2004) 6:69-81.

[32] Meli M.L., Cattori V., Martinez F., Lopez G., Vargas A., Simon M.A., et al., Feline leukemia virus and other pathogens as important threats to the survival of the critically endangered Iberian lynx (Lynx pardinus), PLoS ONE (2009) 4:e4744.
[33] Molia S., Chomel B.B., Kasten R.W., Leutenegger C.M., Steele B.R., Marker L., et al., Prevalence of Bartonella infection in wild African lions (Panthera leo) and cheetahs (Acinonyx jubatus), Vet. Microbiol. (2004) 100:31-41.

[34] Pacitti A.M., Jarrett O., Hay D., Transmission of feline leukaemia virus in the milk of a non-viraemic cat, Vet. Rec. (1986) 118:381-384.

[35] Pedersen N.C., Theilen G., Keane M.A., Fairbanks L., Mason T., Orser B., et al., Studies of naturally transmitted feline leukemia virus infection, Am. J. Vet. Res. (1977) 38:1523-1531.

[36] Rojko J.L., Hoover E.A., Mathes L.E., Hause W.R., Schaller J.P., Olsen R.G., Detection of feline leukemia virus in tissues of cats by a paraffin embedding immunofluorescence procedure, J. Natl. Cancer Inst. (1978) 61:1315-1321.

[37] Rojko J.L., Hoover E.A., Mathes L.E., Olsen R.G., Schaller J.P., Pathogenesis of experimental feline leukemia virus infection, J. Natl. Cancer Inst. (1979) 63:759768.

[38] Rojko J.L., Kociba G.J., Pathogenesis of infection by the feline leukemia virus, J. Am. Vet. Med. Assoc. (1991) 199:1305-1310.

[39] Tandon R., Cattori V., Gomes-Keller M.A., Meli M.L., Golder M.C., Lutz H., Hofmann-Lehmann R., Quantitation of feline leukaemia virus viral and proviral loads by TaqMan $(\mathrm{R})$ real-time polymerase chain reaction, J. Virol. Methods (2005) 130:124-132.

[40] Tandon R., Cattori V., Willi B., Meli M.L., Gomes-Keller M.A., Lutz H., Hofmann-Lehmann R., Copy number polymorphism of endogenous feline leukemia virus-like sequences, Mol. Cell. Probes (2007) $21: 257-266$

[41] Tandon R., Cattori V., Pepin A.C., Riond B., Meli M.L., McDonald M., et al., Association between endogenous feline leukemia virus loads and exogenous feline leukemia virus infection in domestic cats, Virus Res. (2008) 135:136-143.

[42] Torres A.N., Mathiason C.K., Hoover E.A., Re-examination of feline leukemia virus: host relationships using real-time PCR, Virology (2005) 332:272-283.

[43] Vedbrat S.S., Rasheed S., Lutz H., Gonda M.A., Ruscetti S., Gardner M.B., Prensky W., Feline oncornavirus-associated cell membrane antigen: a viral and not a cellularly coded transformation-specific antigen of cat lymphomas, Virology (1983) 124:445-461.

[44] Vogtlin A., Fraefel C., Albini S., Leutenegger C.M., Schraner E., Spiess B., et al., Quantification of feline herpesvirus 1 DNA in ocular fluid samples of clinically diseased cats by real-time TaqMan PCR, J. Clin. Microbiol. (2002) 40:519-523.

[45] Willi B., Boretti F.S., Cattori V., Tasker S., Meli M.L., Reusch C., et al., Identification, molecular characterization, and experimental transmission of a new hemoplasma isolate from a cat with hemolytic anemia in Switzerland, J. Clin. Microbiol. (2005) 43:2581-2585. 